\title{
Digital phase control in a Y-coupled plasmonic terahertz laser system
}

DOI:

10.1109/IRMMW-THz.2012.6380368

Link to publication record in Manchester Research Explorer

\section{Citation for published version (APA):}

Chakraborty, S., Marshall, O., Khairuzzaman, M., Beere, H. E., \& Ritchie, D. A. (2012). Digital phase control in a Ycoupled plasmonic terahertz laser system. In International Conference on Infrared, Millimeter, and Terahertz Waves, IRMMW-THz/Int. Conf. Infrared, Millim., Terahertz Waves, IRMMW-THz https://doi.org/10.1109/IRMMWTHz.2012.6380368

\section{Published in:}

International Conference on Infrared, Millimeter, and Terahertz Waves, IRMMW-THz|Int. Conf. Infrared, Millim., Terahertz Waves, IRMMW-THz

\section{Citing this paper}

Please note that where the full-text provided on Manchester Research Explorer is the Author Accepted Manuscript or Proof version this may differ from the final Published version. If citing, it is advised that you check and use the publisher's definitive version.

\section{General rights}

Copyright and moral rights for the publications made accessible in the Research Explorer are retained by the authors and/or other copyright owners and it is a condition of accessing publications that users recognise and abide by the legal requirements associated with these rights.

\section{Takedown policy}

If you believe that this document breaches copyright please refer to the University of Manchester's Takedown Procedures [http://man.ac.uk/04Y6Bo] or contact uml.scholarlycommunications@manchester.ac.uk providing relevant details, so we can investigate your claim.

\section{OPEN ACCESS}




\title{
Digital Phase Control in a Y-Coupled Plasmonic Terahertz Laser System
}

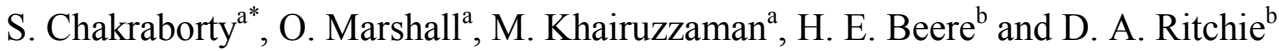 \\ ${ }^{a}$ School of Electrical and Electronic Engineering, University of Manchester, UK \\ ${ }^{\mathrm{b}}$ Cavendish Laboratory, Department of Physics, University of Cambridge, UK \\ *Email: s.chakraborty@manchester.ac.uk
}

\begin{abstract}
Electrically independent terahertz quantum cascade lasers in a $Y$ configuration are coupled via the evanescent electromagnetic field in their shared substrate. Switchable lasing mode solutions are defined in one laser by a longitudinal computer generated hologram introduced to it's plasmonic waveguide. When operated together, this control is extended to the coupled, unmodified laser.
\end{abstract}

\section{INTRODUCTION AND BACKGROUND}

G ROWING interest in $\mathrm{THz}$ technologies has led to a demand for cheaper and more highly functional sources than those in widespread use today. Terahertz quantum cascade lasers (QCLs) are a leading contender, offering milliwatts of optical power from compact, electrically driven, semiconductor devices. However, certain aspects of THz QCL performance have proven difficult to control. In particular, manipulation of single-mode emission frequency and phase has required complex experimental arrangements ${ }^{1}$. The recent development of discretely tunable THz QCLs with embedded longitudinal computer generated holograms (LCGHs) offers an alternate solution, with electronically controlled, single section devices ${ }^{2}$. In contrast to conventional transverse CGHs which give only directional control over incident light, LCGHs provide high resolution digital control in wavevector (k-) space and may therefore be used for photonic engineering. In the search for ever greater functionality we may take inspiration from well established, shorter wavelength laser systems, where straight ridge waveguides have in many cases been replaced with guided wave interconnects, such as waveguide couplers and arrayed waveguide gratings (AWGs), introducing extra degrees of on-chip phase and frequency control. A basic building block of such systems is the $\mathrm{Y}$ coupling of two lasers. Here we integrate the LCGH-QCL approach with a Y-coupled laser architecture in a step toward on-chip, all electronic, THz QCL emission control, offering the prospect of mechanically stable, high speed emission switching without the need for complex external optics or device arrays.

\section{DEVICE ARCHITECTURE AND TESTING}

Coupling in the Y-system is achieved by exploiting the optical mode profile in the QCLs' surface plasmon waveguides. Fig. 1a shows a cross-sectional fundamental mode intensity profile for a single unperturbed semi-insulating surface plasmon (SI-SP) QCL ridge waveguide. It reveals a

This work was supported by EPSRC First Grant EP/G064504/1 and partly supported by HMGCC. (a)
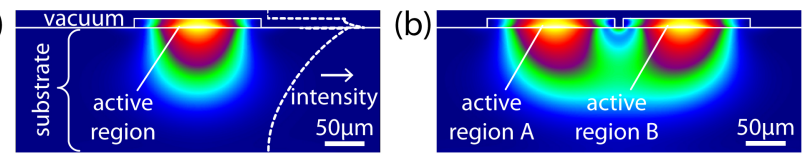

(c)

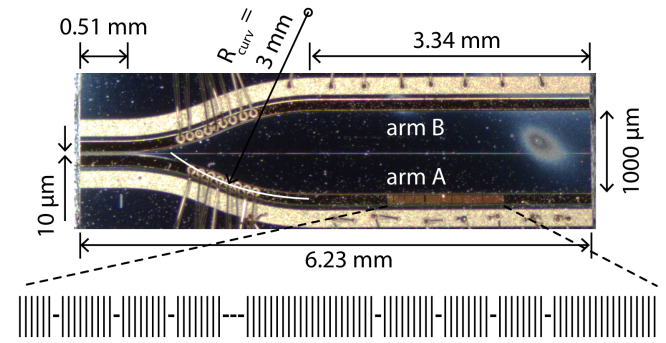

(d)

(e)

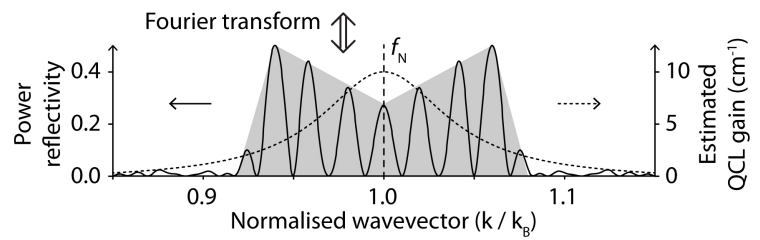

(f)

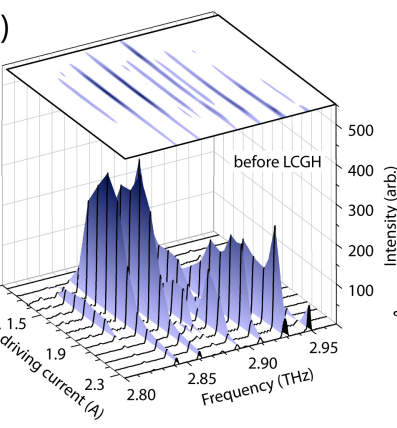

(g)

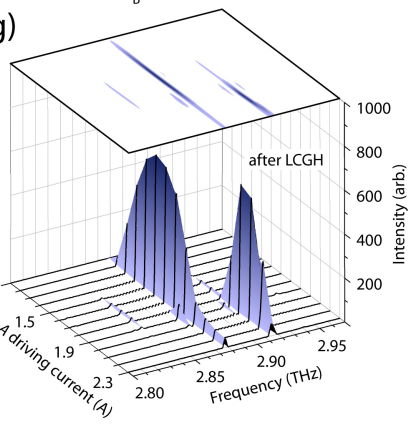

Fig. 1. Simulated cross sectional fundamental mode profiles with (a) a single QCL ridge and (b) two closely spaced ridges. Blue through to yellow represent low to high mode intensities. (c) Photograph of a THz QCL Y system, showing the electrical contacts, wire bonding, LCGH positioning, and critical device dimensions. (d) Symbolic representation of the LCGH. Vertical lines represent milled slits with a minimum spacing $\Lambda$, dashes additional $\Lambda / 2$ lengths. (e) Calculated LCGH spectral power reflectivity (solid line) and its resonance envelope shape (shaded). Multiple resonances occur within the estimated QCL gain bandwidth (dashed line). Emission spectra of arm $A$ (f) prior to, and $(\mathrm{g})$ after the introduction of the LCGH.

significant fraction of the modal power in a large substrate lobe beneath the active region (AR). Coupling between SI-SP ridges in close proximity is possible via this evanescent electromagnetic field, e.g. Fig. 1b. The principal dimensions of the Y-system are shown on the device photograph in Fig. 1c. Contrary to other Y-coupled semiconductor lasers in which waveguides were fully merged, the SI-SP arms share a substrate but their ARs remain physically distinct, with a minimum ridge separation of $10 \mu \mathrm{m}$. Arms were individually 
electrically contacted in an asymmetric two terminal arrangement. Electrical isolation between arms was ensured by the selective post-processing removal of the highly doped GaAs layer between the ARs by focussed ion beam (FIB) milling. QCLs were powered using synchronised pulse generators ( $1 \%$ duty cycle, $1 \mu \mathrm{s}$ pulse length), at $4.5 \mathrm{~K}$. Finally, subsequent to initial device characterisation, the LCGH was introduced directly into the upper metallic waveguide layers of $\operatorname{arm} A$. A symbolic representation of the real-space LCGH structure is given in Fig. 1d. It's spectral reflectivity response (Fig. 1e) was designed to contain multiple Bragg reflectivity peaks intended to harness the underlying coarse tuning of the AR gain to produce switchable single mode laser emission with varying driving current.

\section{RESULTS}

Prior to the introduction of the LCGH, multi-mode emission was observed at almost all driving currents in both arms. The lasing spectra collected from arm $A$ are presented in Fig. 1f. Lasing modes were primarily dictated by the round trip phase solutions of the cleaved Fabry-Pérot (FP) cavities. However, the expected FP mode spacing of $\sim 6.5 \mathrm{GHz}$ for a $6.2 \mathrm{~mm}$ long QCL operating around $2.9 \mathrm{THz}$ was modified by the presence of the waveguide S-bends, which possess their own (unquantified) dispersion characteristics. Distinct spectral changes occurred after the LCGH modification of arm $A$. The LCGHs influence is seen in the redistribution of $\mathrm{THz}$ spectral power in Fig. 1g. The modified arm $A$ displayed switchable single-mode emission at two digitally selected frequencies (2.88 and $2.92 \mathrm{THz}$ ), each corresponding to and originating from an LCGH reflectivity peak (Fig. 1e). Small traces of power are also observed at a third LCGH defined frequency of $2.84 \mathrm{THz}$, though this mode never becomes dominant. In contrast, when operated singly the emission from the unmodified arm $B$ remained multi-moded, even after introduction of the LCGH to $\operatorname{arm} A$. Emission spectra collected from $\operatorname{arm} B$, operated alone between lasing threshold and maximum output power, are presented in Fig. 2a. Clear evidence of optical coupling and LCGH control is present when both arms are operated simultaneously. Fig. 2b shows the measured spectral performance collected under almost identical conditions to Fig. 2a, but with the addition of a constant driving current in $\operatorname{arm} A\left(\mathrm{I}_{\mathrm{A}}\right)$ of $1.8 \mathrm{~A}$, such that it lased at $2.88 \mathrm{THz}$. At low arm $B$ driving current $\left(\mathrm{I}_{\mathrm{B}}\right)$, only the stray emission from $A$ is detected. As $\mathrm{I}_{\mathrm{B}}$ is increased, the former multi-mode emission is suppressed, optical power instead appearing at the three LCGH defined frequencies previously observed in $A$ alone; by far the largest rise in power occurring at $2.88 \mathrm{THz}$. As confirmation that the increased modal power originates from $B$, measurements were repeated with the collection optics aligned to the isolated facet of $A$ (Fig. 2c). Negligible power variation is seen at $2.88 \mathrm{THz}$, with reduced power increases at 2.84 and $2.92 \mathrm{THz}$. In effect, optical coupling between the arms allows the high modal power in the LCGH modified arm $A$ to seed the lasing in $\operatorname{arm} B$, thereby transferring the LCGH phase solutions to the unmodified QCL. One might expect a similar result using a uniform grating, with a single resonance frequency. The advantage of the LCGH approach is that it enables the creation of multiple, finely spaced, reflectivity resonances. As shown, this design flexibility was used to produce switchable emission in $\operatorname{arm} A$. The key consequence is that this switchable

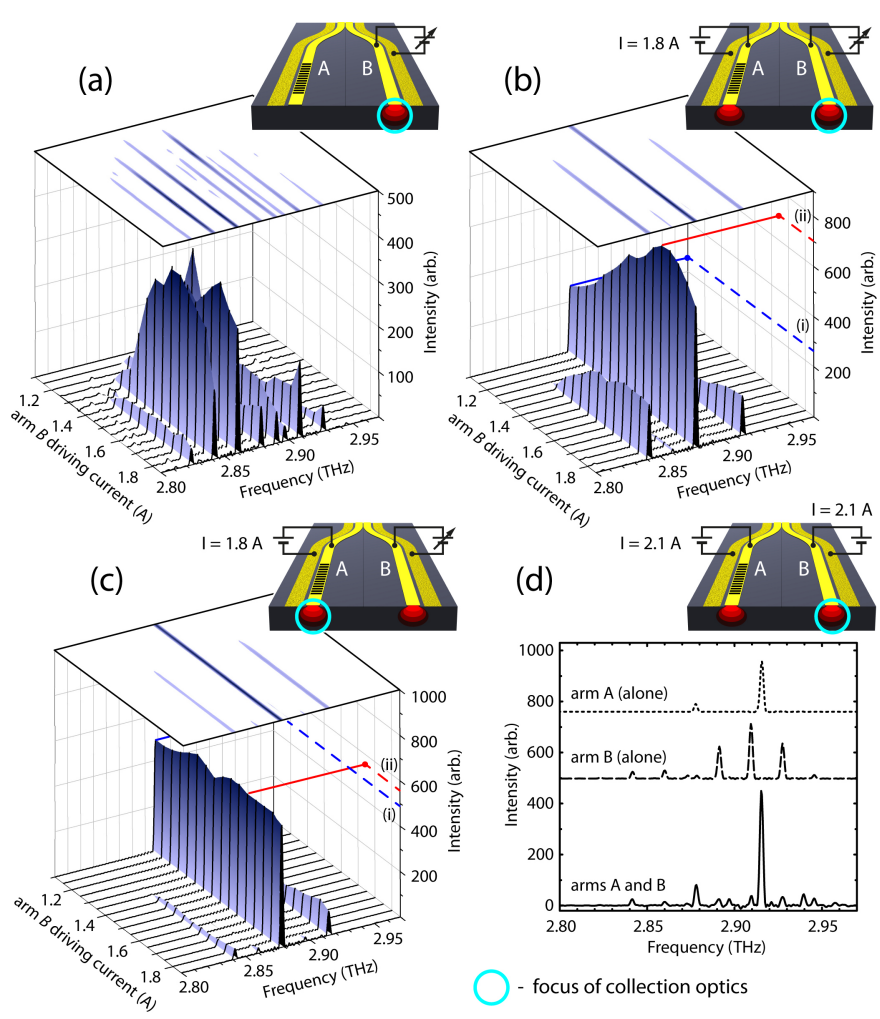

Fig. 1. Y system emission spectra. (a) Collected from arm $B$ alone, between lasing threshold and maximum output power. (b) Collected from the facet of arm $B$ across the same driving current range, with arm $A$ driven at a constant $1.8 \mathrm{~A}$. Modal power at $2.88 \mathrm{THz}$ rises from 260 to 700 (arbitrary units) between points (i) and (ii), a 167\% increase. (c) Collected from the facet of arm $A$, under the same system driving conditions. Modal power at $2.88 \mathrm{THz}$ only rises by $14 \%$ between points (i) and (ii). (d) Collected from the facet of arm $B$, at high QCL driving currents in each arm alone and in combination. Insets depict the device architecture, electrical powering arrangement and focal point of collection optics.

control may also be transferred between $\mathrm{Y}$ arms. Fig. $2 \mathrm{~d}$ displays emission spectra of both $A$ and $B$, operated singly and in combination with $\mathrm{I}_{\mathrm{A}}=\mathrm{I}_{\mathrm{B}}=2.1 \mathrm{~A}$. Individually, arm $A$ predominantly lases upon the high frequency LCGH mode at $2.92 \mathrm{THz}$, arm $B$ upon three modes at $\sim 2.89,2.91$ and $2.93 \mathrm{THz}$. Once again, the combined Y system output is LCGH controlled, with the power of $B$ largely constrained to the LCGH mode solution at $2.92 \mathrm{THz}$, evidenced by it's increased power at the expense of the other modes.

\section{CONCLUSION}

Mode switching within a THz QCL is achieved through optical coupling to a second laser on the same substrate, which is digitally controlled by an integrated longitudinal hologram. The transference of photonic control between electrically isolated constituent $\mathrm{THz}$ QCLs in a Y system is a significant step toward the goal of more complex on-chip phase switching and control architectures in active plasmonic terahertz systems.

\section{REFERENCES}

[1] M. S. Vitiello, and A. Tredicucci, "Tunable Emission in THz Quantum Cascade Lasers”, IEEE Trans. Terahertz Sci. Tech. 1, 76-84, 2011.

[2] S. Chakraborty, O. Marshall, M. Khairuzzaman, C.-W. Hsin, H. E. Beere, and D. A. Ritchie, "Longitudinal Computer Generated Holograms for Digital Frequency Control in Electronically Tunable Terahertz Lasers", http://arxiv.org/abs/1205.3042 